\title{
Peer-Reviewed Science and the Specialty of Pediatric Neuropsychology
}

\author{
Cecil R. Reynolds ${ }^{1}$
}

Received: 4 March 2019 / Accepted: 4 March 2019 / Published online: 30 April 2019

(C) American Academy of Pediatric Neuropsychology 2019

As recently as the early twentieth century, conventional scientific wisdom was that children were "just little adults", and no scientific research was needed to study children as distinct from adults. Clearly, the conventional scientific wisdom has changed and the need to conduct scientific research on children is very clear. Children's brains are extremely complex, and due to their rapid rates of development, a moving target and much additional research on the best assessment and treatment practices for children are urgently needed. In fact, the mature or adult brain, denoting a fully functioning brain with completion of the myelination and pruning processes and a fully functioning prefrontal cortex (along with its communication circuitry) does not occur on average until after the age of 21 years, reaching into the early to middle stages of the third decade of life.

An important goal is the continued differentiation of pediatric from adult practice and continuing to establish the scientific basis of this differentiation - children more so than adults are moving targets, with more rapidly changing brains who develop in decidedly nonlinear fashions. Children are also dependent upon adults to seek out and make them available for pediatric neuropsychological services. The developmental trajectory and dependency of the pediatric population are continuously ripe topics for research related to services not only needed but how best to provide them in these contexts.

Just as clinical child and adolescent psychology are specialty areas of practice, distinct from but related to the practice of clinical psychology, the American Academy of Pediatric Neuropsychology (AAPdN), professional sponsor of the Journal of Pediatric Neuropsychology (JPNP), exists because pediatric neuropsychology is likewise a specialty area of practice, distinct from but related to clinical neuropsychology. Consistent with one of its key missions, to advance training

Cecil R. Reynolds

crrh@earthlink.net

1 Texas A\&M University, College Station, TX, USA and education in pediatric neuropsychology, the AAPdN established JPNP just over 5 years ago in partnership with the longstanding scientific publisher, Springer, a publisher well-known to most neuropsychologists. In keeping with the mission of the AAPdN, JPNP solicits and publishes peerreviewed works that advance education and practice in pediatric neuropsychology. While basic science in the field is accepted, applied science is preferred; however, the journal publishes a variety of works that are consistent with the mission of the AAPdN, including reviews of literature that provide a synthesis of sound work of others that conclude with new insights, especially into improving delivery and quality of clinical services, grand rounds consisting of one or more case studies that likewise provide new insights into practice and clinical services, and special issues or sections devoted to matters of importance in the field wherein substantive matters may remain unresolved in the science and practice of pediatric neuropsychology. All such papers should have the same ultimate goal however - the improvement of clinical services or their delivery to persons ages 0-21 years. In all such matters, new science and new insights take precedence.

Having been a journal editor now for more than 23 years, having served as an editor for other professional associations, including the American Psychological Association and the National Academy of Neuropsychology among others, I am aware of the politics of science, but also of more recent assaults by predatory journal publishers on the integrity of scientific publishing.

The integrity of pediatric neuropsychological science is critical to providing clinical services that are actually effective in improving the care and lives of our patients. Likewise, the quality of our science is critical to delivering effective services as well. To this end, ensuring the integrity and quality of work in JPNP, all papers (except archival documents of the AAPdN and clearly so designated), including this editorial, are subjected to peer review. In general, the growth, which has been nearly explosive over the last three decades, of neuropsychology can be attributed to the continuing growth of the science 
of brain-behavior relationships that underlies practice in clinical neuropsychology and putting this science to work in patient care. Good science benefits from peer review and determining what is and is not effective in serving patients depends upon science - effective clinical practice is not an art - it is the application of science to solving patient problems and improving life outcomes. Additionally, the AAPdN Board of Directors and Springer publishing, the publisher of JPNP, both provide various levels of oversight regarding the direction of the journal and its mission and success in accomplishing these goals. Those familiar with scientific publishing will recognize Springer as a longstanding, highly reputable purveyor of scientific publications in the form of books and scientific journals as well.

The journal is open to all, and I would like to extend a personal invitation to researchers and practitioners to develop and share their work in these various formats with the universe of practitioners and other researchers who are involved in providing pediatric neuropsychological services. The journal holds no prejudice and seeks only high quality work that contributes to the missions of the AAPdN, especially in its role as one aspect of the Academy's advancement of training and education in pediatric neuropsychology and improvement of patient care in this chronically under-served population.

Children reside in systems - schools, families, and sometimes legal and governmental placements (e.g., juvenile facilities), acute care and rehabilitation facilities, and others. There remains a need for expanding access and quality of pediatric neuropsychological care in all such settings where children are found, and I welcome work on what services are needed where and how best to deliver such services effectively. Each year, the National Institutes of Mental Health publishes a list of the most underserved populations of persons in the USA, and children have made the top 10 in every year.
The complexity of new models and demands on clinical practice and the myriad populations to which they must be applied also lead us to stress to the research community that to break new ground one must lay a strong foundation for multi-disciplinary research, a foundation that must include the careful development of cultural and systemic contexts in each domain of clinical practice and in research findings in submissions to the journal.

I am honored to have been recommended and subsequently chosen to lead what I see as an outstanding group of Associate Editors and Consulting Editors in this effort to promote the scientific basis of practice in our chosen profession. I am also adding two special sections to the journal, one devoted to research in pediatric behavioral neuroimaging under the leadership of Dr. Jesse Bledsoe, and a Grand Rounds section under the leadership of Dr. Grace Mucci. Knowing all of the affiliated Associate Editors and their work, the success of Journal of Pediatric Neuropsychology in promoting the advancement of all aspects of pediatric neuropsychological practice will stem from their efforts, and any failing will be mine for not taking full advantage of their talents and dedication to the profession.

With this issue, you will begin to see the earliest fruits of our collective efforts. If you are involved in research with implications for pediatric neuropsychological science and practice, as broadly defined and as practiced, Journal of Pediatric Neuropsychology is interested in your work. Any and all recommendations for improvements to the journal or our editorial process are welcome as well and can be sent directly to me.

Publisher's Note Springer Nature remains neutral with regard to jurisdictional claims in published maps and institutional affiliations. 